\title{
Jugular Foramen Tumors
}

\author{
C Rayappa \\ Senior Consultant, Department of Head and Neck and Skull base Surgery, Apollo Speciality Hospital, Chennai, Tamil Nadu, India
}

Correspondence: C Rayappa, Department of Head and Neck and Skull Base Surgery, Apollo Speciality Hospital Chennai-600040, Tamil Nadu, India, e-mail: drrayappa@yahoo.com

\section{ABSTRACT}

J ugular foramen tumors are rare cranial base lesions that present diagnostic and management difficulties. Paragangliomas were the most frequent lesions, followed by schwannomas and meningiomas. These tumors have characteristic radiological features. Radical resection of these tumors with preservation of the lower cranial nerves is the treatment of choice. Despite the advances in skull base surgery, new postoperative lower cranial nerve deficits still represent a challenge.

Keywords: J ugular foramen, Glomus jugulare, Schwannoma, Meningioma, Infratemporal fossa type-A, Petro-occipital trans-sigmoid approach.

\section{INTRODUCTION}

The jugular foramen region is a complex area of the skull base and important neurovascular structures vizjugular bulb, lower cranial nerves and internal carotid artery are present in this region. Several types of tumors occur in this area and these tumors are deeply located and involve these important neurovascular structures and bone at the skull base.

A variety of tumors occur in this region and the most common tumor arising from this area is paraganglioma. Other frequent neoplasms in this region are schwannomas and meningiomas. Since 1997, we have surgically managed 48 cases of jugular foramen tumors in our hospital. Of these, 27 were glomus jugulare, 15 were schwannomas, three were meningiomas, two were chondroblastomas and one case of plasmocytoma.

The paragangliomas arise from glomus tissue. In the temporal bone, these are found in the adventitia of the jugular bul $b$ beneath the floor of middle ear, adjacent to the tympanic branches of the glossopharyngeal and vagus nerves and in the bone of the promontory, close to the mucosal lining of the middle ear. ${ }^{1}$ In most patients with glomus jugulare, hearing loss and pulsatile tinnitus are the early presenting symptoms. Patient develops weak voice and dysphagia due to paralysis of IX and $X$ cranial nerves when the tumor enlarges.

It has been hypothesized that jugular foramen schwannomas may originate from the ganglia of cranial nerves IX and $X$, which are situated close to the jugular foramen. Following the path of least resistance, these tumors may expand superiorly into the posterior fossa cistern, or inferiorly into the parapharyngeal space. ${ }^{2}$ J ugular foramen schwannomas can present with various symptoms depending on the size and the anatomic location of the tumor.
Progressive lower cranial nerve deficits represent the most frequent presenting complaints. The second most common clinical picture mimics the presentation of a cerebellopontine angle lesion with ipsilateral sensorineural hearing loss, tinnitus and vertigo.

Primary jugular foramen meningiomas likely arise from ectopic arachnoid granulations or pacchionian bodies associated with the lower cranial nerves. ${ }^{3}$ They first invade the hypotympanum, infralabyrinthine air cell tracts and middle ear. Simultaneous intracranial and extracranial extension into the cervical spaces is common. M eningiomas arising from the adjacent areas may secondarily invade the jugular foramen. Meningiomas of the jugular foramen typically spread between individual cranial nerve filaments and into the sheaths surrounding the nerves at the skull base. ${ }^{4}$ Jugular foramen meningiomas usually present with weak voice and dysphagia due to involvement of lower cranial nerves.

$V$ arious other lesions arise from this region vizchondrosarcomas, chondroblastomas, petrous bone cholesteatoma, etc. These tumors differ significantly in their vascularity, aggressiveness, pattern of spread and invasion of nearby structures. Each of these pathology should be dealt with individually because of the diversity in the tumor behavior.

Collaborative effort of the otolaryngologists, neurosurgeons and interventional neuroradiologists has improved the diagnosis as well as management of jugular foramen tumors. The objective of treatment is radical resection with preservation of the cranial nerves and vessels.

\section{SURGICAL ANATOMY}

The jugular foramen is an anatomically complex region of the cranial base and understanding the complex surgical anatomy of this region is necessary to gain adequate surgical 


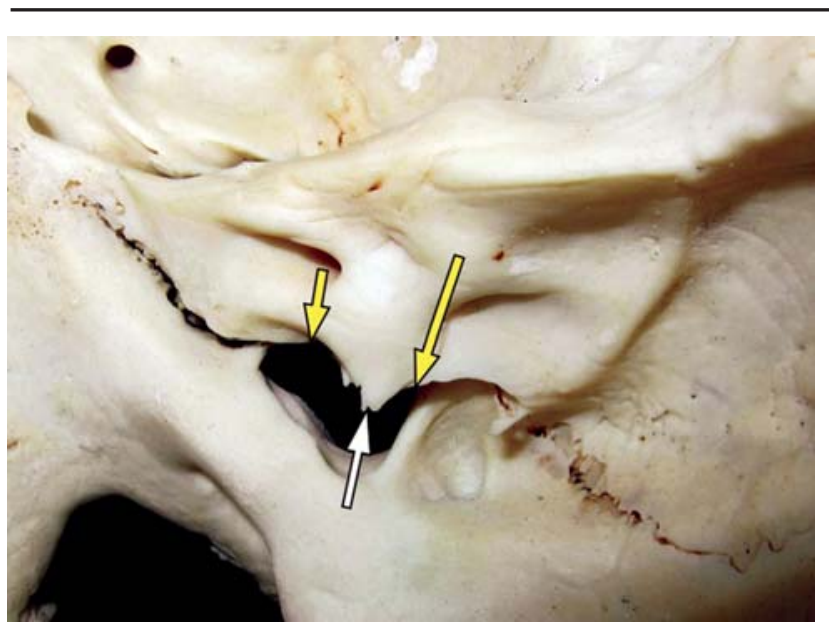

Fig. 1: Endocranial view of the jugular foramen on right side. Jugular spine (white arrow) partially divides the jugular foramen into pars nervosa (small yellow arrow) and pars venosa (long yellow arrow)

exposure. The jugular foramen lies at the junction of the base of the temporal and occipital bones. In 1934, Hovelaque ${ }^{5}$ described the jugular foramen as having two compartments (Fig. 1), i.e. an anteromedial "pars nervosa" containing the 9th cranial nerve and the inferior petrosal sinus and a posterolateral "pars venosa" containing the 10th and 11th cranial nerves, the jugular bulb and the posterior meningeal artery. O ccasionally, a fibro-osseous septum can be found between the jugular spine of the temporal bone and that of the occipital bone, separating the jugular foramen into these two compartments. M ore often, however, there is no septation and the jugular foramen exists as one compartment. The 9th cranial nerve enters the jugular foramen medially and is the most anterior of the cranial nerves. A s it exits the skull base, it is positioned as the most anterior and lateral of the nerves. This lateral position makes it slightly more susceptible to injury during resection. The 11th cranial nerve exits the skull base as the most posterior and medial of these nerves. The 10th cranial nerve exits medially between the ICA and the internal jugular vein. A ccording to Sen et al, the cranial nerves lie anteromedial to the jugular bulb and maintain a multifascicular histoarchitecture (particularly the 10th cranial nerve). ${ }^{6}$ The vagus nerve contains multiple fascicles and the glossopharyngeal and accessory nerves contain one and two fascicles respectively. Glomus tumors of the temporal bone as well as meningiomas can invade the cranial nerve fascicles, and infiltration of these nerves can occur despite normal function. In these situations, total resection may not be possible without sacrifice of these nerves. The inferior petrosal sinus (IPS), which often consists of multiple channels, courses from the cavernous sinus and empties into the medial aspect of the jugular bulb. The position of the lower cranial nerves with respect to the IPS is variable. The IPS will often pass between the 9th nerve anteriorly and the 10th and 11th nerves posteriorly. Therefore, overpacking the IPS or cautery in this area can cause nerve injury. The tympanic branch of the glossopharyngeal nerve (J acobson's nerve) and the auricular branch of the vagus nerve (A rnold's nerve) cross the jugular foramen. The internal carotid artery runs medial to the tympanic bone and gives off the carotidtympanicum branches.

\section{RADIOLOGICAL EXAMINATION}

The most important radiological differential diagnoses of the jugular foramen tumors include paragangliomas, vestibular schwannomas and meningiomas. High resolution computed tomography (HRCT) with bone windows and the MRI with gadolinium are essential and complimentary investigations to accurately del ineate the extent of the tumor preoperatively and optimize its management. CT scan is useful for analysis of bony structures, tumor calcification and hyperostosis or bone destruction. The normal jugular foramen has smooth cortical borders surrounded by cancellous bone. Jugular foramen schwannomas appear isodense or slightly hyperdense on CT scans and usually cause enlargement of the jugular foramen with well-defined intact cortical bony margins and no signs of bony infiltration. In contrast, paragangliomas and meningiomas cause irregular erosion of the margins of the jugular foramen and infiltrates surrounding bone (Fig. 2). Unlike jugular paragangliomas, bone destruction of the jugular fossa is much less evident in meningiomas. The caroticojugular spine is characteristically eroded in paragangliomas, whereas mild hyperostosis may be present in meningiomas.

Schwannomas are usually iso- or hypointense on T1weighted images and iso- or hyperintense on T2-weighted images and enhance with gadolinium (Fig. 3). On MRI, paragangliomas appear hypointense on $\mathrm{T}$ 1-weighted images, whereas on T2-weighted images the tumor component is isointense. A fter gadolinium administration, paragangliomas show dishomogenous enhancement with a salt-and-pepper appearance and multiple flow void areas (Fig. 4). On M RI, meningiomas are iso- or hypointense on $\mathrm{T} 1$ and hyperintense on T2 images. The meningiomas can be distinguished from schwannomas by the presence of irregular borders, enhancing more intensely with gadolinium and, most often, a lower intensity on T2. The flow voids characteristic of paragangliomas is absent in the meningiomas (Figs $5 \mathrm{~A}$ and $B$ ). There frequently encountered dural tail sign after gadolinium administration is pathognomonic of meningiomas. Schwannomas tend to compress the jugular bulb but paragangliomas and meningiomas invade the vessel with intraluminal growth (Fig. 6). The specific imaging criteria that may help predict the status of the cranial nerves are the intradural extension and the configuration of the medial border of the tumor at the jugular foramen. When 


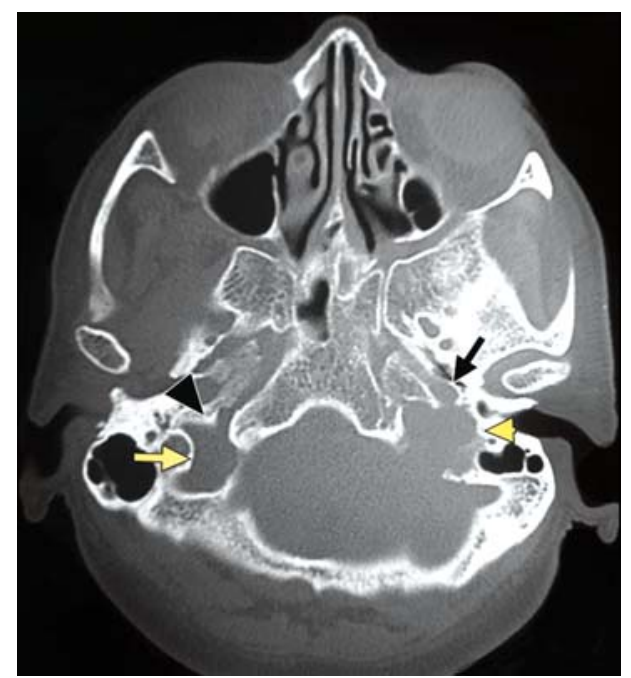

Fig. 2: Axial CT scan of skull base shows normal jugular foramen (yellow arrow) with intact cortical margin and the jugular spine (black arrow head) on left side. On the right side, the cortical margin is lost (yellow arrowhead) because of the infiltrating glomus jugulare. Black arrow points at the carotid canal, the posterior wall of which is also eroded by the tumor

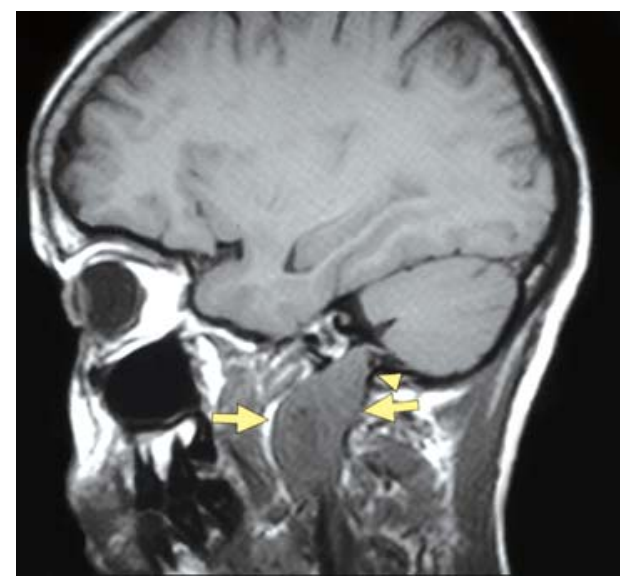

Fig. 3: MRI shows the jugular foramen schwannoma extending into the neck (yellow arrows). The cortex of the jugular foramen is intact (yellow arrowhead)

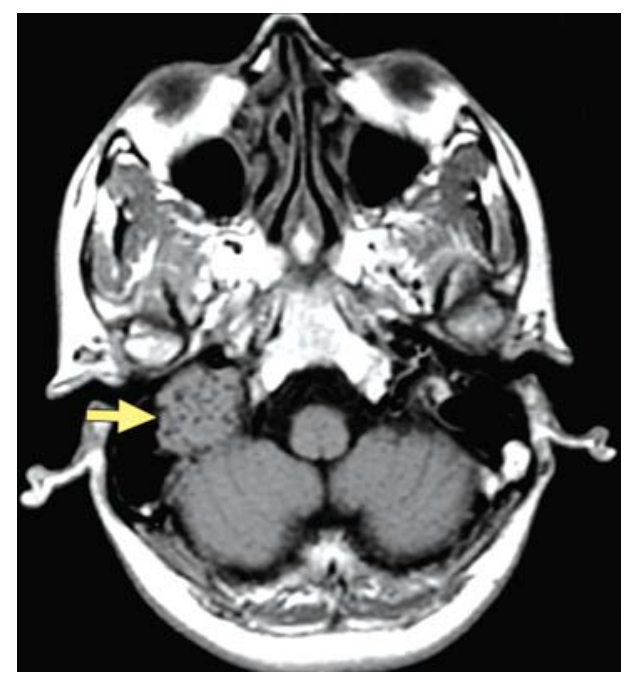

Fig. 4: MRI shows jugular foramen lesion with salt-and-pepper appearance (yellow arrow) produced by the flow void of the intratumoral vessels, typical of glomus jugulare
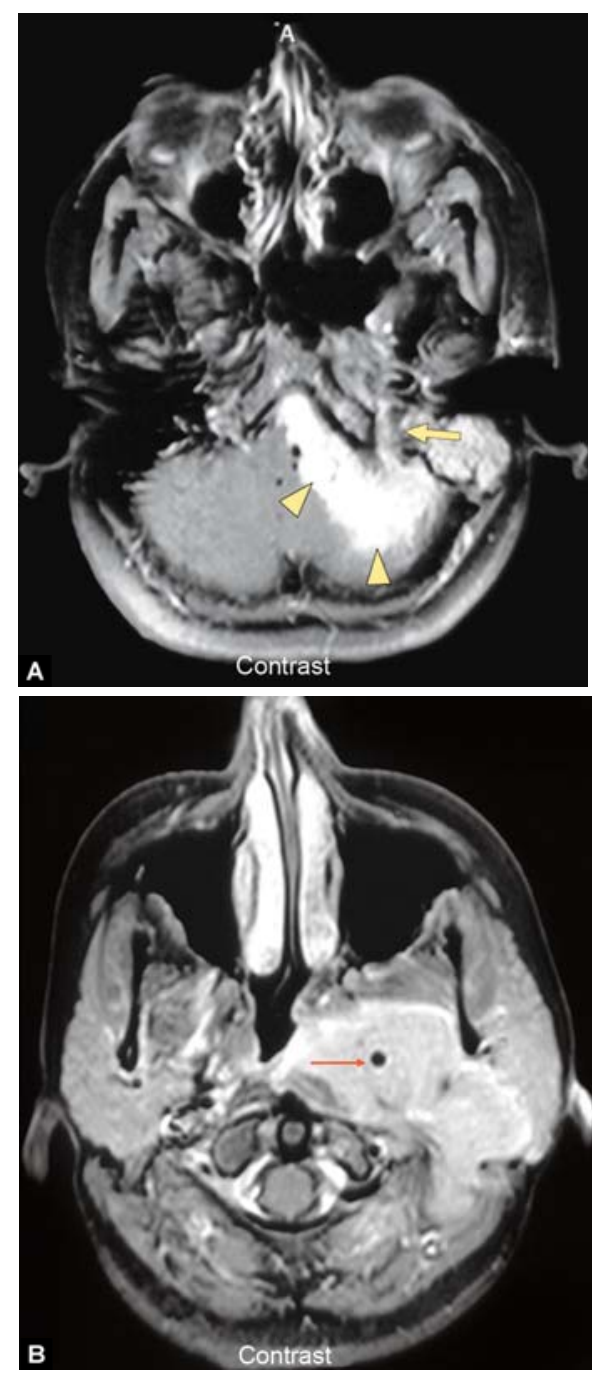

Figs 5A and B: Primary jugular foramen meningioma. (A) Axial contrastenhanced T1-weighted MR image shows a left jugular foramen meningioma (yellow arrow) with a large basal cistern component (arrowhead) that spreads along the dura. (B) MR image below the skull base shows a large extracranial component of the tumor encircling the ICA (red arrow)

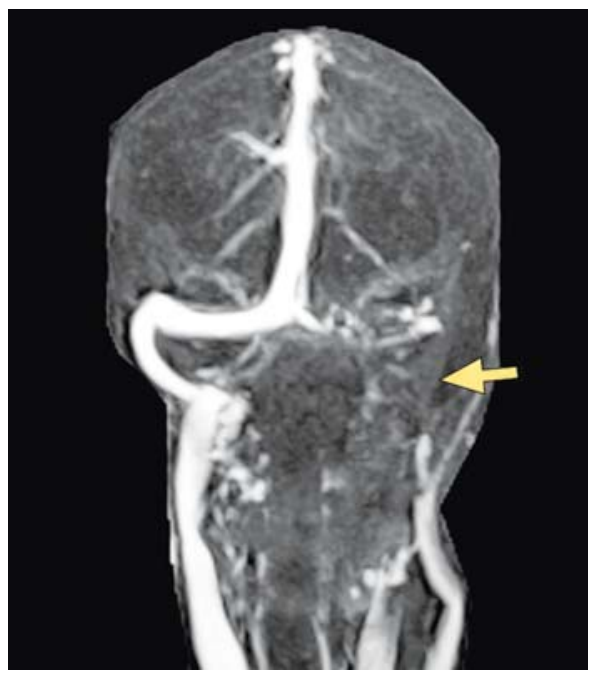

Fig. 6: MR image shows absence of blood flow through the sigmoid sinus (yellow arrow) 
the tumor extends intradurally, it tends to insinuate itself among the delicate fascicles of these nerves. W hen the M RI and $\mathrm{CT}$ scans demonstrate that the medial border of the tumor is indistinct, there is a high likelihood that the tumor has infiltrated the cranial nerve fascicles in the jugular foramen. ${ }^{7}$

Digital subtraction angiography is routinely performed when the lesion in the jugular foramen is vascular (Fig. 7). This study helped to find the bleeding vessels and the relationship with the internal carotid and vertebral artery. This study is performed 2 to 3 days prior to the date of surgery and the hypervascular tumor is embolized (Fig. 8) to reduce the intraoperative bleeding. When the tumor is surrounding the internal carotid artery, temporary balloon occlusion test is performed to evaluate the cross circulation through the circle of Willis. If there is no neurol ogical deficit during the temporary balloon occlusion test, permanent

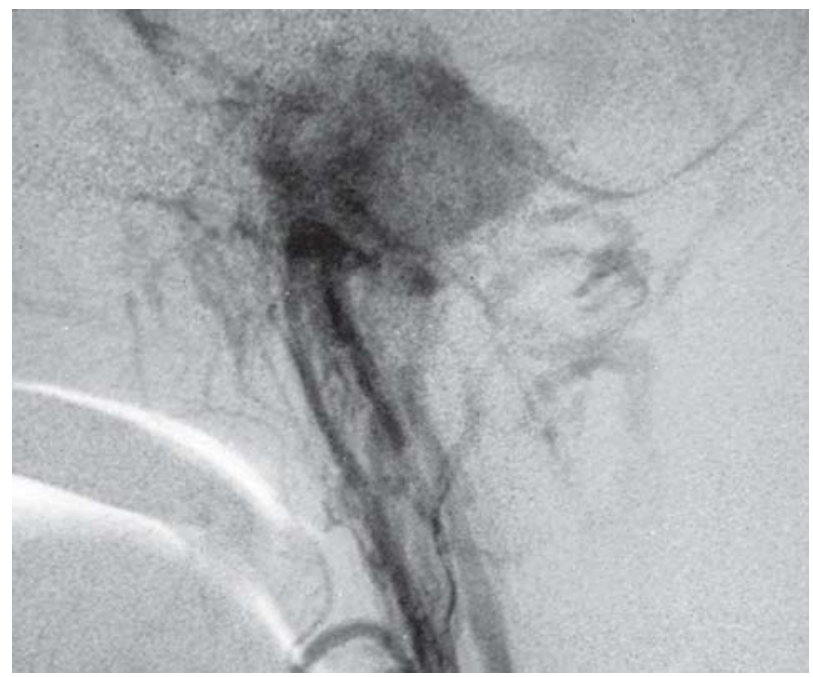

Fig. 7: Preoperative DSA showing tumor blush at the site of jugular foramen

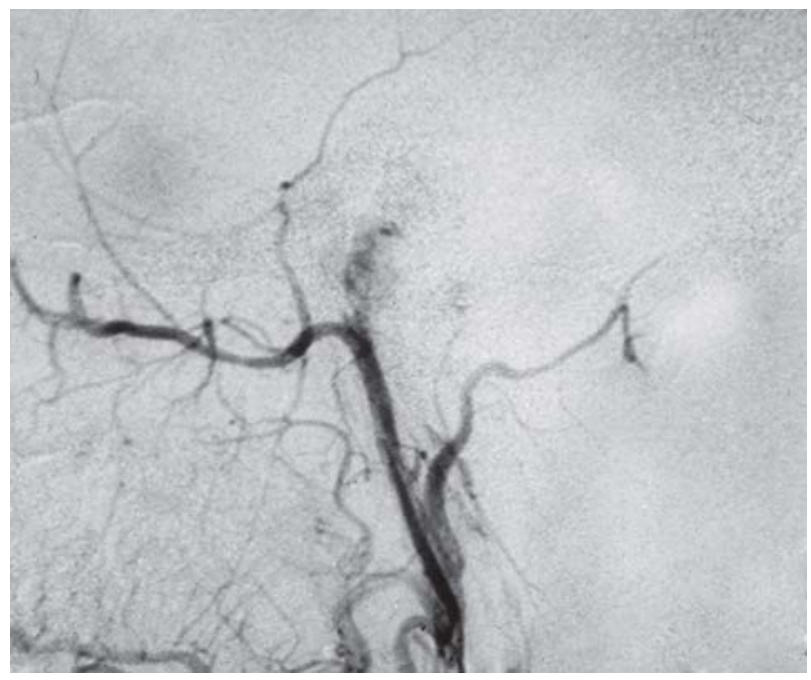

Fig. 8: Postembolization DSA shows no tumor blush occlusion of the ICA is performed using a balloon or microcoil (Fig. 9). Then the internal carotid artery can be sacrificed without any neurological deficits.

\section{SURGERY}

Total tumor removal is the treatment of choice for jugular foramen tumors. The ideal surgical approach should adhere to the basic principles of obtaining a wide access to the whole tumor with minimal brain manipulation while minimizing morbidity related to the additional cranial nerve deficits. A dditional goals of the surgery include preservation of inner ear, middle ear and the facial nerve function.

Paragangliomas usually infiltrate the carotid canal and may invade the adventitia of the internal carotid artery (ICA). For this reason, the surgical approach needs to control the whole length of the artery to be able to perform a subadventitial dissection of the invaded artery. Despite the excellent exposure of the jugul ar foramen area and the ICA, the infratemporal fossa type-A (ITF-A) approach has the disadvantages of the postoperative conductive hearing loss resulting from the ear canal closure and facial paresis related to the anterior rerouting of the facial nerve. ${ }^{8}$

On the contrary, schwannomas erode but do not infiltrate the petrous bone. U nlike paragangliomas, schwannomas do not tend to infiltrate the internal carotid artery walls. Preservation of hearing and facial nerve functions appeared to be the specific advantages of the petro-occipital transsigmoid (POTS) approach. ${ }^{9}$ Sigmoid sinus transection gives optimal visualization of the cerebellopontine angle, whereas opening the jugular bulb provides adequate control of the jugular foramen.

M eningiomas are often intimately entwined with lower cranial nerves. Therefore, subtotal resection is necessary if

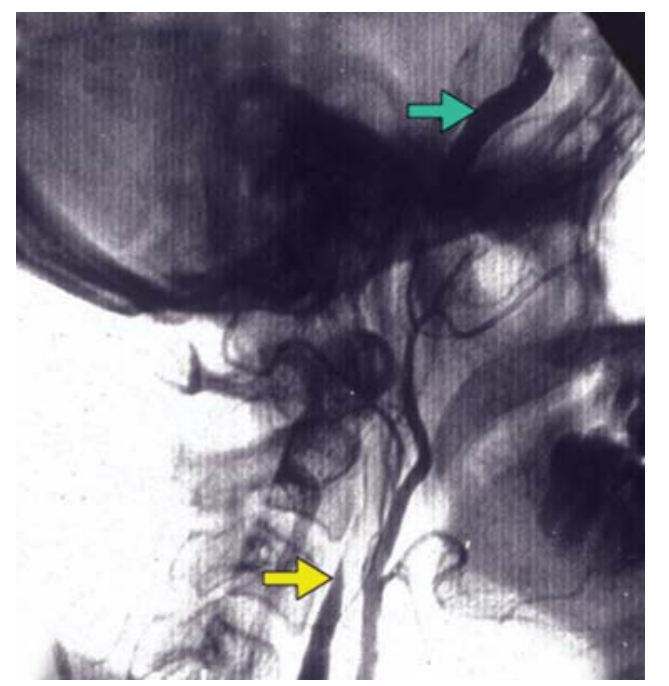

Fig. 9: Permanent occlusion of ICA with contrast-filled balloon in cavernous sinus (green arrow). Absence of blood flow in the distal part of ICA (yellow arrow) 
cranial nerve preservation is desired. On the other hand, resection of involved cranial nerves is associated with a lower chance of tumor recurrence. When the cranial nerve dysfunction is present prior to surgery, the decision to resect the affected cranial nerves is straightforward. However, when the cranial nerve dysfunction is absent or mild, prior to surgery, the decision of resecting the cranial nerves is difficult because of the morbidity associated with multiple lower cranial nerve dysfunction. A combined transtemporal/ transcranial/transcervical approach will be needed for those extensive tumors with temporal bone involvement with intracranial and cervical extension. Infratemporal fossa type-A approach of Fisch is capable of this exposure when combined with retrolabyrinthine craniotomy. If severe or profound hearing loss is present, the intracranial component may be managed by translabyrinthine approach. For larger and more anteriorly based lesions, a transcochl ear approach may be used. Extensive meningiomas with a large intracranial component in association with extensive temporal bone invasion may require a total petrosectomy approach to obtain sufficient exposure.

Sudden palsy of previously functioning lower cranial nerves in elderly patients would be met with serious morbidity. Watchful expectancy is therefore adopted, and surgery is only indicated in case of cranial nerve deficits and/or impending neurologic complications.

\section{Infratemporal Fossa Type-A}

Patient is placed in the supine position with the head turned $45^{\circ}$ to the opposite side. Care was taken not to compress the opposite jugular vein. A sickle shaped incision was made from the temporal region to the cricoid level curving $5 \mathrm{~cm}$ behind the pinna. Skin flap is raised superficial to the plane of temporal is fascia and sternomastoid muscle. Rectangular shaped, anteriorly based [attached to the posterior wall of cartilaginous external auditory canal (EAC)] myoperiosteal flap was raised over the mastoid (Fig.10A). The EAC was divided at the junction of the cartilage and bony EAC, medial to the attachment of the myoperiosteal flap. Skin of the cartilaginous EAC was dissected, everted and then sutured (Fig.10B). Second layer closure was done using the myoperiosteal flap to prevent cerebrospinal fluid (CSF) fistula.

The temporalis muscle was reflected anteriorly. Sternomastoid muscle was detached from mastoid and the muscle was dissected along its anterior border. The major vessels of the neck (common carotid artery, internal and external carotid arteries and internal jugular vein) and lower cranial nerves (IX, X, XI and XII) are dissected (Fig.10C). $V$ ertebral artery is identified at the skull base after dissection of the lateral process of $\mathrm{C} 1$ and the superior and inferior oblique muscles.

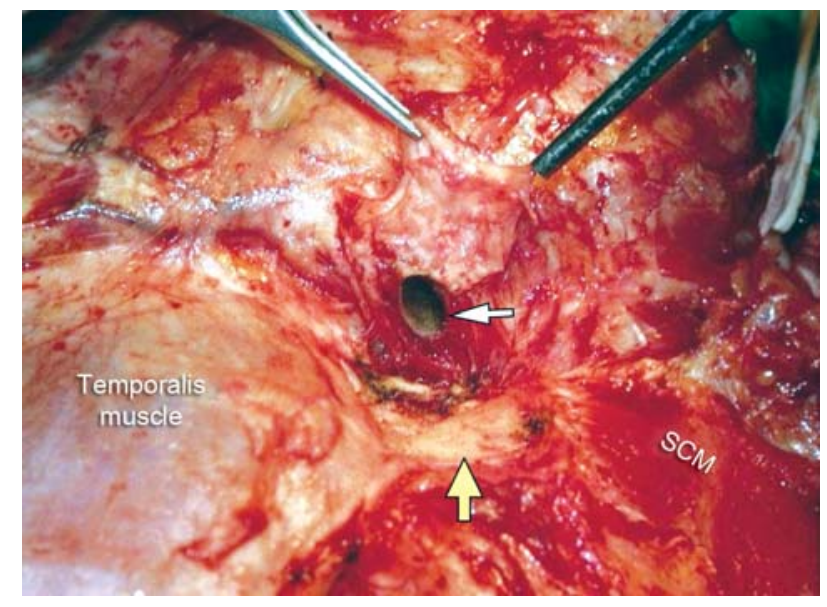

Fig. 10A: Anteriorly based myoperiosteal flap attached to posterior part of cartilaginous EAC (held by the forceps). White arrow points at the divided EAC. Yellow arrow points at mastoid. SCM—sternocleidomastoid muscle

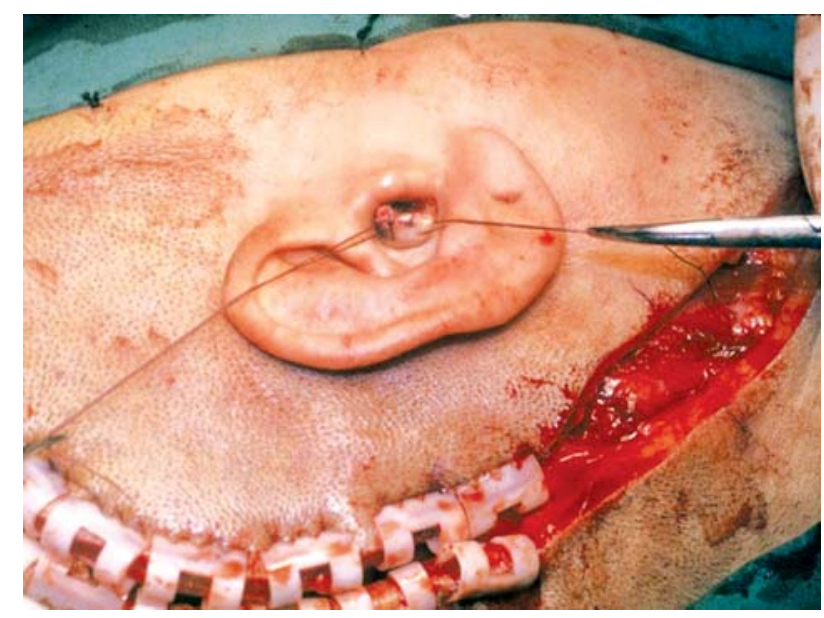

Fig. 10B: Blind closure of EAC. Everted canal skin being sutured

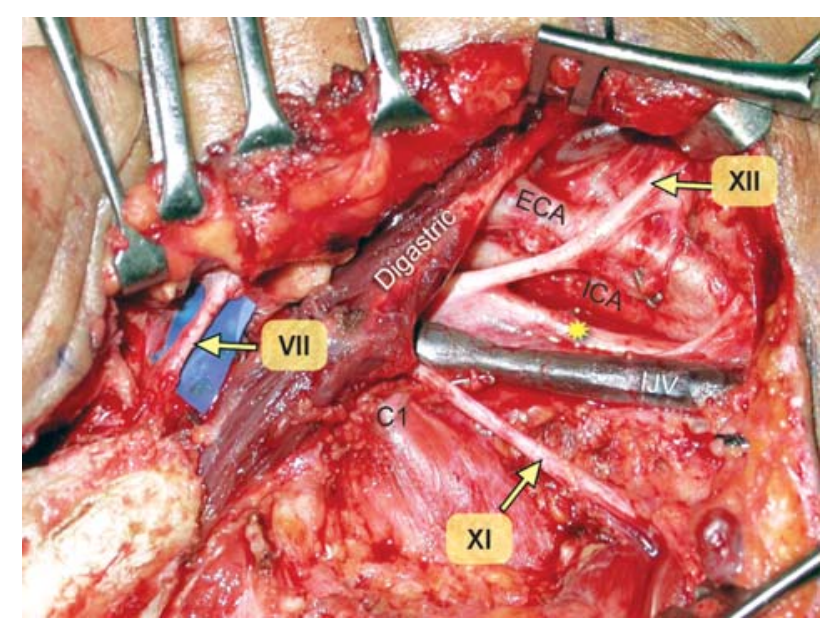

Fig. 10C: Neck dissection. VII - facial nerve; XI - spinal accessory nerve; Star - vagus nerve; XII - hypoglossal nerve; $\mathrm{C} 1$ - transverse process of C1 vertebra; ECA - external carotid artery; ICA - internal carotid artery; IJV - internal jugular vein 
The facial nerve is dissected in the stylomastoid and parotid region. This nerve bisects the angle formed by the tympanic bone and posterior belly of digastric muscle. The nerve is dissected beyond its bifurcation in the parotid, which helps to reduce the traction during anterior rerouting. In smaller tumors, the nerve need not be rerouted and the tumor can be removed by working anterior and posterior to the fallopian canal (fallopian bridge technique). ${ }^{10}$ This technique avoids the postoperative facial palsy, which occurs in almost all patients in whom the nerve was rerouted. When the nerve is infiltrated by the tumor, the nerve is resected and grafted.

Subtotal petrosectomy is done. Entire sigmoid sinus is skeletonized, presigmoid posterior fossa dura is dissected and the retrofacial cells are removed. Facial nerve was decompressed (Fig. 10D) from geniculate ganglion to the stylomastoid foramen and then rerouted anteriorly (Fig. 10E). Tumor within the middle ear, eustachian tube and mastoid air cells are removed using bipolar cautery. Removal of the tympanic bone and styloid process exposes the internal carotid artery. Paragangliomas usually infiltrate the carotid canal and may invade the adventitia of the internal carotid artery. For this reason, the surgical approach needs to control the whole length of the artery to be able to perform a subadventitial dissection of the invaded artery.

The next step is excision of the jugular bulb. Before that, the blood flow through the sigmoid sinus has to be stopped. Bone over the sigmoid sinus is removed. Dural incision is made anterior as well as posterior to the sigmoid sinus and then the sinus is ligated. The author prefers to stop the blood flow by extraluminal packing (between the bone and the sigmoid sinus) with Surgicel. Internal jugular vein is ligated in the neck and then the jugular bulb and the tumor were excised (Fig. 10F). Glomus tumors usually do not involve the medial wall of the sigmoid sinus and the bulb. There will be brisk bleeding from the openings of the inferior petrosal sinuses in the medial wall of the bulb. This bleeding can be controlled by packing these openings with Surgicel. It is difficult to identify the cranial nerves in the jugular foramen. Only way to safeguard these nerves is to identify them distally and then traced proximally towards the jugular foramen.

The removal of an intradural component at the same stage is not safe because connecting the subarachnoid space with the neck space during tumor removal would create a high risk of postoperative CSF leak. Following strategy should be followed in these cases. Small tumors $(<1 \mathrm{~cm})$ are removed at the same stage and the dura is closed with a muscle plug. Staged removal is performed in larger tumors $(>1 \mathrm{~cm})$. Extradural part is removed first, followed by a second-stage POTS approach for the intradural part. ${ }^{11}$

Most patients with jugular foramen schwannoma and meningioma have normal hearing and facial nerve function.

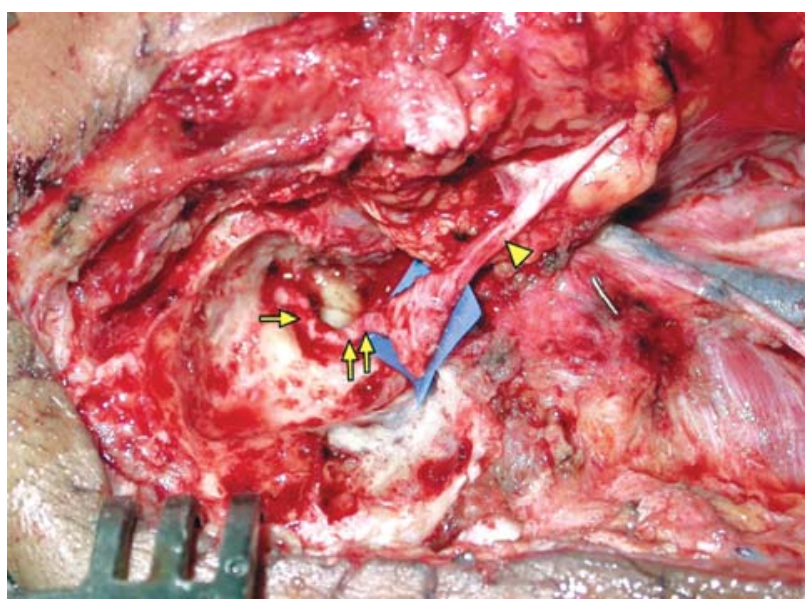

Fig. 10D: Facial nerve is decompressed and elevated out of fallopian canal from the area of geniculate ganglion to the stylomastoid foramen. Tympanic segment (single arrow), mastoid segment (double arrow) and the extratemporal segment (arrowhead) are seen

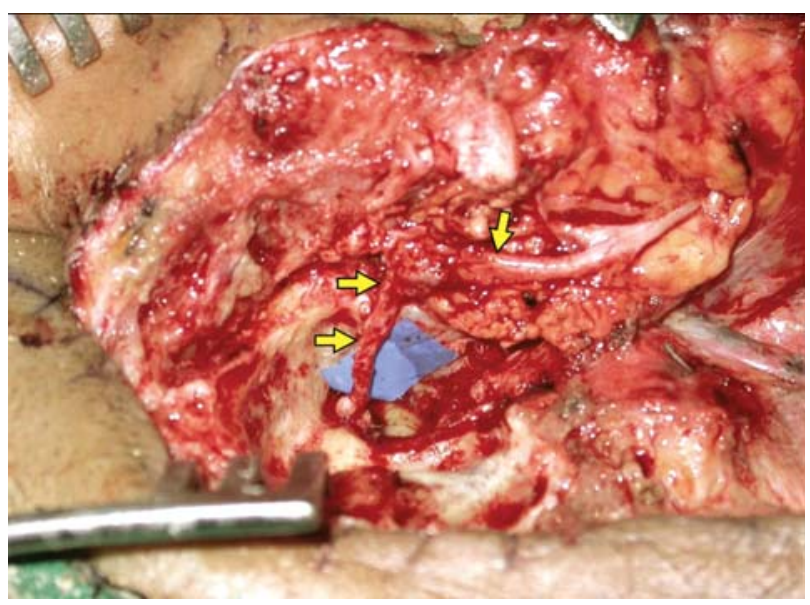

Fig. 10E: Anteriorly rerouted facial nerve (arrows)

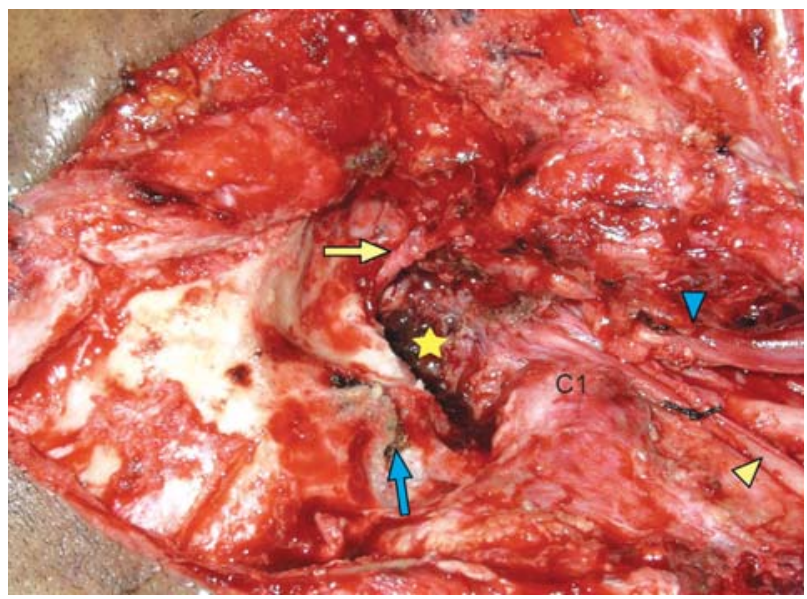

Fig. 10F: Glomus jugulare tumor was excised completely with the jugular bulb after extraluminally blocking the sigmoid sinus (blue arrow) and ligating the IJV (blue arrowhead). Anteriorly rerouted facial nerve (yellow arrow), spinal accessory nerve (yellow arrowhead), lateral process of $\mathrm{C} 1$ vertebra $(\mathrm{C} 1)$ and the jugular foramen area from where the tumor was excised (yellow star) are seen 


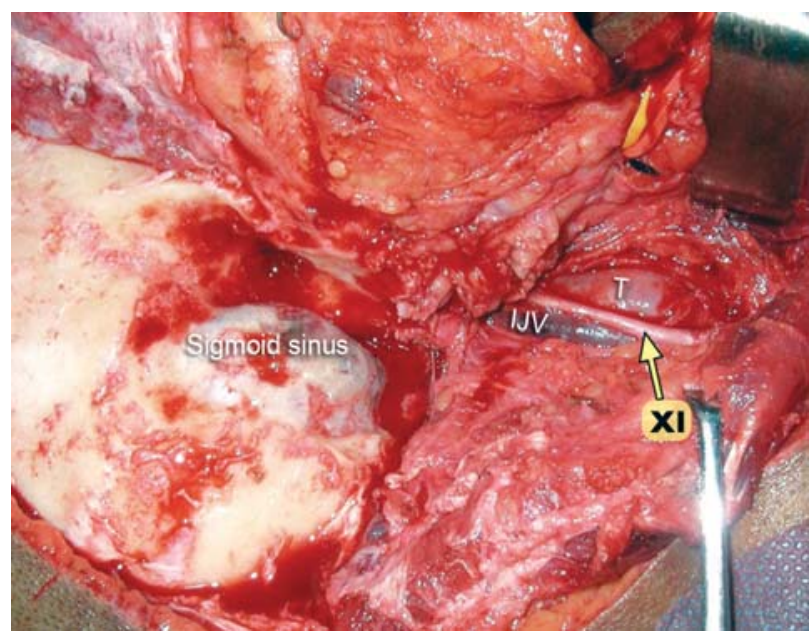

Fig. 11A: An extended mastoidectomy is performed. The sigmoid sinus is skeletonized and retrosigmoid craniectomy is performed. Upper neck is explored. Internal jugular vein (IJV), spinal accessory nerve (XI) and the cervical part of the tumor are seen

The surgeon has to choose an approach that preserves hearing and facial nerve function without hampering adequate tumor visualization and control.

The POTS offers direct access to the jugular foramen area, cerebellopontine angle, and upper cervical region with preservation of the middle and inner ear without the need for facial nerve transposition, as is required by the infratemporal fossa type-A approach.

This approach is designed primarily for nonvascularized lesions and it offers a direct and conservative route to the jugular foramen and adjacent areas while keeping the facial nerve in place and preserving the external auditory canal and middle ear. A reas that can be controlled by this approach include the jugular foramen, cerebellopontine angle, occipital condyle, the ipsilateral lower clivus, the vertical portion of internal carotid artery lying below the eustachian tube, and the jugulocarotid space. This approach entails a retrolabyrinthine petrosectomy combined with the retrosigmoid craniotomy and occlusion of the sigmoid sinus and jugular vein.

\section{Petro-Osseous Trans-Sigmoid (POTS) Approach}

A C-shaped postauricular skin incision is made. It begins approximately $3 \mathrm{~cm}$ above the auricle, curves 4 to $5 \mathrm{~cm}$ behind the postauricular sulcus, and ends $2 \mathrm{~cm}$ below the tip of the mastoid. A skin flap was raised and retracted anteriorly. The sternocleidomastoid muscle is retracted posteriorly. Blunt dissection anterior to lateral process of the atlas reveals the internal jugular vein. A $n$ extended mastoidectomy is performed. The sigmoid sinus is skeletonized and the mastoid tip is amputated. The bone overlying the skel etonized sigmoid sinus and sinodural angle is removed. A piece of bone overlying the sigmoid sinus is

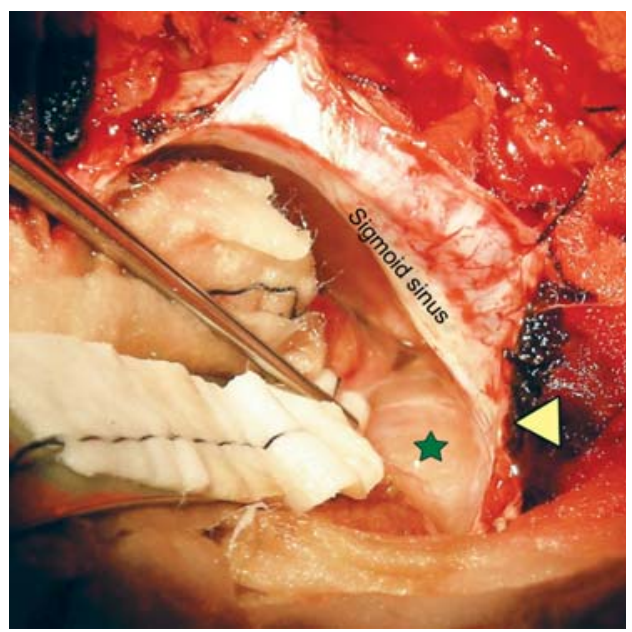

Fig. 11B: The dura was opened at the retrosigmoid area and then the dural incision was extended anteriorly after dividing the sigmoid sinus (yellow arrowhead) exposes the intracranial portion of the tumor (green star)

left in place to allow extraluminal compression with Surgicel. The dura is separated from the underlying bone. The retrofacial cells were then drilled. The endolymphatic sac and the duct are identified and sectioned to allow further detachment of the dura from the labyrinth. The infralabyrinthine cells were drilled taking care not to injure the cochlea lying superolaterally (Fig. 11A ). The proximal part of the sigmoid sinus is compressed extraluminally with a piece of Surgicel placed between the sinus and overlying bone at its junction with the lateral sinus. Theinternal jugular vein in the neck is ligated. N ext the sigmoid sinus is closed proximally and distally with silk ties and the dura is opened with the knife. The posterior fossa dura is opened with a horizontal incision starting approximately $3 \mathrm{~cm}$ posterior to the sigmoid sinus, coursing anteriorly, transversing the sinus, and ending at the level of the posterior semicircular canal (Fig. 11B). The dura is held by two stay sutures, one for the superior dural flap and the other for the inferior one. Tumor removal proceeds accordingly. All opened labyrinthine and the retrofacial cells should be sealed with bone wax to prevent CSF rhinorrhea. The retrosigmoid dura is approximated with interrupted $4-0$ vicryl sutures. A small defect at the level of the jugular foramen remains. The defect as well as the operative cavity is plugged with abdominal fat.

Total removal should be aimed at. However, in elderly patients with normal lower cranial function, when the tumor is found to be invading the lower cranial nerves, some surgeons advocate leaving a small piece of tumor around the infiltrated nerve to avoid significant postoperative morbidity.

Radiation therapy is recommended by some authors to treat paragangliomas because it is associated with lower morbidity of lower cranial nerves. ${ }^{12}$ This treatment is 
indicated when the patient refuses surgery, poor general condition, carotid artery invasion with insufficient collaterals and in elderly patient $>65$ years with intact lower cranial nerves preoperatively. The beneficial effects of radiation therapy for paragangliomas of temporal bone remains, however, uncertain. ${ }^{13}$ The effect of irradiation appears to be on the blood vessels and fibrous elements of the lesion. ${ }^{14,15}$ M ore than $80 \%$ tumor control is reported after radiation therapy. Radiosurgery using gamma knife or a linear accelerator system may be more beneficial in reducing side effects of irradiation. Radiation-induced neoplasm has been described after radiotherapy for benign glomus jugulare tumors. ${ }^{16}$

\section{COMPLICATIONS}

L ower cranial nerve pal sies are perhaps the most significant causes of morbidity in cranial base operations and proper steps must be taken before the onset of serious secondary complications.

Patients with pre-existing lower cranial nerve problems usually have had time for the normal side to compensate and do not have a significant problem with the airway or swallowing postoperatively. If vagus nerve injury or sacrifice occurs during surgery, paralysis of the pharynx, palate and the vocal cord will ensue. This results in weak voice and significant aspiration. Performing a tracheostomy is preferable to repeated bouts of aspiration and it provides access for good pulmonary toilet. It can make the recovery phase of the patient much smoother. In most cases, the initial breathiness and aspi ration of liquids gradually resolve when the contralateral vocal cord compensates. However, if this compensation is inadequate, complete glottic closure can be reestablished by surgically medializing the paralyzed vocal cord (type I thyroplasty). A rytenoid adduction can be combined with a type I thyroplasty if sufficient posterior closure is not obtained with an implant alone. M aintenance of adequate nutrition is important and a feeding gastrostomy is more comfortable and easier to manage than nasogastric tubes. The patient must be reassured that these are temporary measures and can be removed when sufficient recovery has taken place. Vocal cord medialization improves glottic closureand decreases aspiration risk. B ut the sensory deficits produced by denervation of the afferent pathways, poor coordination of swallow and weakness with resultant pooling still result in significant symptoms. Thus, patients with lower cranial neuropathies remain at risk for aspi ration, even after the glottic insufficiency has been corrected. A $n$ experienced speech-language pathologist can assist with the monitoring of the patient, recommend diet modifications, and provide intensive swallowing therapy. ${ }^{17} \mathrm{Crico}$ pharyngeal myotomy is performed during the operative procedure to improve transit to the cervical esophagus and prevent aspiration by spillover into the larynx. A n occasional patient may have severe intractable aspiration requiring laryngotracheal seperation.

Palatal paralysis can cause nasal regurgitation as well as change in the quality of speech because of velopharyngeal incompetence. Unilateral palatal adhesion (palatopexy) is indicated for patients with permanent unilateral palatal paralysis that does not respond to conservative measures. ${ }^{18}$ A $n$ adhesion is surgically created at the level of Passavant's ridge, a site of normal closure of the velopalatine valve. This procedure affixes the soft palate to the posterior wall of nasopharynx and this improves the quality of speech and stops nasal regurgitation.

V oice and swallowing therapy should be considered a complimentary treatment regardless of any other interventions.

Spinal accessory nerve injury results in shoulder pain and restricted range of motion because of the paralysis of sternomastoid and trapezius muscles. A ggressive postoperative physical therapy can prevent the development of adhesive capsulitis and scapul ar "winging", al though normal shoulder range of motion and strength are not possible. ${ }^{19}$

CSF leak is a common problem after any skull base surgery. CSF leak to the neck is particularly difficult to manage as a result of the impossibility of applying any sort of compression. A ll preventing steps to be taken to minimize the risk of a CSF leak vizproper dural closure, sealing of all opened infralabyrinthine cells with bone wax and obliteration of the operated cavity with long strips of abdominal fat. If the risk of leak is high, CSF pressure may be lowered in the postoperative period using continuous lumbar drainage.

\section{REFERENCES}

1. Lattes R, Waltner JG. Nonchromaffin paraganglioma of the middle ear. Cancer 1949;2:447.

2. Von Doersten PG. Tumors of the ear and temporal bone. Philadel phia: W illiams and W ilkins 2000:374-87.

3. Thompson SW, Cass SP. M eningioma of the jugular foramen. In: Jackler RK, Driscoll CLW (Eds). Tumors of the ear and temporal bone. Philadelphia: Lippincott: Williams and W ilkins 2000;361-73.

4. Nager GT, Heroy J, Heoplinger M . M eningiomas invading the temporal bone with extension to the neck. A m J Otolaryngol 1983;4:297-324.

5. Hovelaque A. Osteologie. Paris, G Doin and Cie 1934;2: 155-56.

6. Sen C, Hague K, Kacchara R, Jenkins A, Das S, Catalano P. Jugular foramen: Microscopic anatomic features and implications for neural preservation with reference to glomus tumors involving the temporal bone. Neurosurgery 2001;48: 838-48.

7. Macdonald AJ, Salzman KL, Harnsberger HR, Gilbert E, Shelton C. Primary jugular foramen meningioma: Imaging appearance and differentiating features. A J R 2004;182:373-77. 
8. Fisch U. Infratemporal fossa approach to tumors of the temporal bone and base of the skull. J Laryngol Otol 1978;92:949-67.

9. Mazzoni A, Sanna M. A posterolateral approach to the skull base: The petro-occipital trans-sigmoid approach. Skull Base Surg 1995;5:157-67.

10. Pensal ML, Jackler RK. Removal of jugular foramen tumors: The fallopian bridge technique. Otolaryngol Head Neck Surg 1997; 117:586-91.

11. Sanna $M$, et al. Decision making in skull base surgery, in Sanna $M$, et al (Eds): A tlas of microsurgery of the lateral skull base. Thieme Medical Publishers 2008;330-35.

12. Larner J M, Hahn SS, Spaulding CA, Constable W C. Glomus jugulare tumors: $L$ ong-term control by radiation therapy. Cancer 1992;69:1813-17.

13. Green JD, B rackmann DE, N guyen CD, A rriaga M A, Telischi $F F$, Dela Cruz A. Surgical management of previously untreated glomus jugulare tumors. L aryngoscope 1994;104:917-21.
14. Brackmann DE, House WF, Terry R, Scanlan RL. Glomus jugulare tumors: Effect of irradiation. Trans A m A cad Ophthalmol Otolaryngol 1972;76:1423-31.

15. Springate SC, W eichsel baum RR. Radiation or surgery for chemodectoma of the temporal bone: A review of local control and complications. Head Neck 1990;12:303-07.

16. Lalwani AK, Jackler RK, Gutin PH. Lethal fibrosarcoma complicating radiation therapy for benign gl omus jugulare tumor. A m J Otol 1993;14:398-402.

17. Carrau RL, Murry T. Evaluation and management of adult dysphagia and aspiration. Current Opinion in O tolaryngology and Head and Neck Surgery 2000;8:489-96.

18. Netterville JL, Civantos FJ. Rehabilitation of cranial nerve deficits after neurotologic skull base surgery. Laryngoscope 1993;103 (Suppl 60):45-54.

19. Roberts WL. Rehabilitation of the head and neck cancer patient. In: M CG arvey $C L$ (Ed). Physical therapy for the cancer patient. New Y ork: Churchill Livingstone 1990;47-65. 